\title{
Diastereomeric Amides Derived from Malonic Acid: the Role of Chiral Auxiliaries and of the Nature of Co-Acids in the Mixed Kolbe Electrolyses
}

\author{
Marilia O.F. Goulart ${ }^{\mathrm{a}}$, and Hans-Yürgen Schäfer ${ }^{\mathrm{b}}$ \\ ${ }^{a}$ Departamento de Química, Centro de Ciências Exatas e Naturais, Universidade \\ Federal de Alagoas, 57072-970 Maceió - Al, Brazil \\ ${ }^{\mathrm{b}}$ Organisch-Chemisches Institut der Universität, Corrensstrasse 40, \\ D-48149, Münster, Germany
}

Experimentos visando o acoplamento diastereosseletivo de mono-amidas do ácido metilmalônico, sintetizadas a partir de aminas quirais comerciais [S-(+)-1-ciclo-hexiletilamina e (R)-(+)1-feniletilamina] foram realizados, utilizando-se oxidação anódica de Kolbe [cela não dividida, Pt (ânodo e cátodo), $\mathrm{MeOH}$, neutralização de 5\% a 10\% com solução metanólica de $\mathrm{KOH}(1 \mathrm{M}), 200$ a $250 \mathrm{~mA} / \mathrm{cm}^{2}$ ], usando diferentes co-ácidos (ácidos hexanóico, trimetilsililacético, dietilfosfonoacético e ftaloilglicina).

Amidas de cadeia longa ou sililadas (dímeros mistos de Kolbe) foram obtidas com bom rendimento (56 a 63\%) e baixa diastereosseletividade, na presença de excesso de co-ácidos, em conjunto com produtos derivados de caminhos alternativos, principalmente produtos de desproporcionamento e derivados metoxilados.

$\mathrm{O}$ acoplamento radicalar mostrou-se altamente sensível à natureza dos radicais envolvidos, sendo mais efetivo entre radicais de reatividade oposta. Radicais eletrofílicos acoplam entre si em muito pequena extensão.

Acoplamentos diastereosseletivos, na presença dos auxiliares quirais acíclicos, não foram expressivos.

Eletrólise de Kolbe realizada com a ftaloilglicina forneceu dímeros simétricos, N-metoximetilhidroxilactama e N-metoxiftalimida. Na presença de ácido hexanóico, além dos produtos já citados, foram obtidas uma imida alquilada e a hidroxilactama correspondente.

Experiments towards the diastereoselective coupling of new malonic acid amides synthesized with commercially available chiral amines $[(\mathrm{S})-(+)-1$-cyclohexylethylamine and $(\mathrm{R})-(+)-1$ phenylethylamine] as chiral auxiliaries were performed through Kolbe oxidations [undivided cell, $\mathrm{Pt}$ (anode and cathode), $\mathrm{MeOH}, 5 \%$ to $10 \% \mathrm{KOH}$ neutralisation, 200 to $250 \mathrm{~mA} / \mathrm{cm}^{2}$ ], using different co-acids (hexanoic, trimethylsilylacetic and diethylphosphonoacetic acids and phthaloylglycine).

New long chain and silylated amides (mixed Kolbe dimer) were obtained in good yields (56 to $63 \%$ ) and low diastereoselectivity, in the presence of a large excess of co-acids, together with disproportionation and non-Kolbe $(\mathrm{nK})$ products, mainly methoxy derivatives.

Coupling was more effective with radicals with opposite reactivity. Electrophilic radicals couple between themselves to a lesser extent.

Diastereoselective induction was not high.

Studies performed with phthaloylglycine, under Kolbe conditions, in the absence and presence of hexanoic acid as a co-acid led to the symmetrical dimer, a N-methoxymethyl-hydroxylactam and a N-methoxyphthalimide. In the presence of hexanoic acid, the latter products were obtained together with alkylated imide and the corresponding hydroxylactam.

Keywords: diastereoselective Kolbe reaction, malonamic acids, phthaloylglycine, hydroxylactam 


\section{Introduction}

Radical chemistry provides mild reaction conditions for the formation of C-C bonds. Anodic decarboxylation of carboxylic acids is an useful synthetic method for generating radicals (Kolbe electrolysis) or carbocations (nonKolbe electrolysis) (Scheme 1). The generated radicals can be used in homo- and heterocouplings or in addition to double bonds ${ }^{1}$, being a powerful synthetic tool ${ }^{2-4}$. Yields and selectivities of the Kolbe synthesis are strongly dependent on the structure of the acid and on reaction conditions ${ }^{1}$. High current densities and high carboxylate concentrations favour the formation of dimers, as well as weakly acidic medium. Platinum and methanol are, respectively, the electrode and solvent of choice. Methanol oxidation is inhibited by the formation of the carboxylate layer ${ }^{1,2}$. An important advantage of this method is that functional groups in the carboxylic acid components are tolerated, so there is no need for protection-deprotection reactions. In the presence of co-acid, as the intermediate radicals combine statistically, besides the expected mixed Kolbe dimer, two homocoupling products are also generated. If the less costly acid is used in excess, the number of major products is reduced to two, simplifying the isolation of the cross-coupled compound. Besides Kolbe products, disproportionation of the generated radicals together with reactions from the non-Kolbe pathway (extended oxidation leading to the carbocation) are expected (Scheme 1). It is useful to report that the maximum yield of mixed dimer for Kolbe eletrolysis in the presence of co-acid, in a concentration 10 times greater, is $91 \%{ }^{1}$. To calculate maximum yield, on use $n .100 / 1+n$ where $n=[$ co-acid $] /[\text { acid }]^{1}$. As reported, the yields of Kolbe electrolysis are considered good, even in the range of $34 \%{ }^{4}-43 \%^{2}$. In spite of the relatively low yield, it has important synthetic and industrial applications ${ }^{5}$. Similar radicals produced homogeneously do not couple with useful yields and there is only one example of a metal-based oxidant $\left(\mathrm{OsCl}_{6}{ }^{-}\right)$which gives comparable coupling ${ }^{6}$.

Radical reactions and their stereochemical aspects are of fundamental importance in organic chemistry, and have been exhaustibly studied and are fairly well understood ${ }^{7}$. Control of acyclic stereochemistry of intermolecular radical reactions was recently reviewed ${ }^{8}$. Diastereoselective coupling derived from Kolbe electrolysis is far less studied. High diastereoselectivity was obtained only recently ${ }^{9}$, through the use of 1,4-induction by chiral cyclic amide auxiliaries.

This paper reports an extension of this approach using new derivatives of methylmalonic acids, half substituted with readily available amines with an acyclic stereogenic center. For this study, the chiral auxiliaries were chosen based on their availability and inactivity in electrochemical terms. Earlier studies showed that the easily available, commodity chemicals, (R)-(+)- and (S)-(-)-1-phenylethylamine used as chiral auxiliaries are very valuable in asymmetric synthesis ${ }^{10,11}$ and led to high yield asymmetric reductive amination ${ }^{12}$. Comprehension of the factors governing the stereoselectivity of intermolecular electrogenerated radical coupling of acyclic systems is of further importance.

It is useful to report that few examples of Kolbe electrolysis of amides and imides have been available in the literature, most of them leading to non-Kolbe products ${ }^{1,9,13}$. Anodic methoxylative decarboxylations (Hofer-Moest reaction), related to the non-Kolbe pathway, have been used to generate several interesting nitrogen heterocycles, key intermediates for stereoselective syntheses ${ }^{14}$.

\section{Experimental}

\section{Equipments}

Melting points are uncorrected. Mass spectra were obtained by using an A.E.I. MAT-312, Finnigan and CH-7A, Varian, in conjunction with an SS200 Data Acquisition, Varian; for GC/MS, a GC 1400, Varian and a CH-7A with data system SS200, Teknivent and/or Shimadzu GC 8A. ${ }^{1} \mathrm{H}$ and ${ }^{13} \mathrm{C}$-NMR spectra were measured at a WM 300, Bruker. NMR spectra were obtained by using $\mathrm{CDCl}_{3}$ solvent with TMS reference, unless otherwise stated. IR spectra were measured in $\mathrm{cm}^{-1}$ at an IR-408, Shimadzu and FT-IR, Nicolet. Elemental analyses were performed at M. Beller

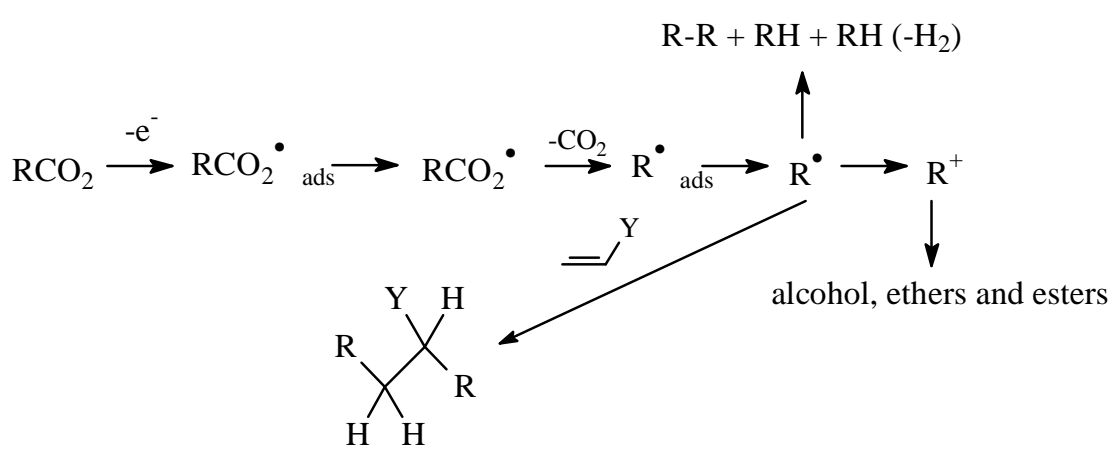

Scheme 1. General mechanism for Kolbe electrolysis. 
Microanalysis Laboratory, in Göttingen. For GC analyses, GC-9A, with Shimadzu C-R3A integrator were used, with the following columns:

- FS-HP1-CB, 25 m, $0.32 \mathrm{~mm}$ internal diameter, $0.25 \mathrm{~m}$ film thickness;

- FS-SE54-CB, $50 \mathrm{~m}, 0.32 \mathrm{~mm}$ internal diameter, $0.25 \mathrm{~m}$ film thickness.

\section{Chemicals}

The chemicals (S)-(+)-1-cyclohexylethylamine (CHA), (R)-(+)-1-phenylethylamine (PEA), trimethylsilylacetic acid, triethylphosphonoacetate, hexanoic acid and diethyl methylmalonate were purchased from Janssen, Aldrich, Merck and Fluka and used without further purification.

\section{Synthesis of N-substituted-2-methylmalonamic acids}

The syntheses followed the general scheme:

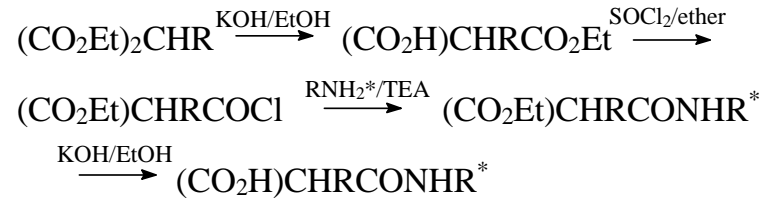

The following procedure is typical for synthesis of the substrates. The amounts are expressed in mol or mmol.

Diethyl methylmalonate $(34.8 \mathrm{~g}, 0.20 \mathrm{~mol})$ in $100 \mathrm{~mL}$ abs. ethanol was added, with stirring, to a solution of of $\mathrm{KOH}(11.7 \mathrm{~g}, 0.21 \mathrm{~mol})$ in $150 \mathrm{~mL}$ abs. ethanol. The solution was left overnight, and the $\mathrm{pH}$ measured (7-8). The white precipitate (di-potassium salt) was filtrated $(2.94 \mathrm{~g}$, $0.015 \mathrm{~mol}, 7.5 \%$ ) and the resulting solution evaporated under reduced pressure. The semisolid opaque residue was dissolved in $20 \mathrm{~mL}$ of water and extracted twice with 20 $\mathrm{mL}$ of petroleum ether. The organic phase was concentrated, leading to colourless oil $(3.13 \mathrm{~g}, 0.017 \mathrm{~mol})$, the starting ester. Acidification with $\mathrm{HCl}$ until $\mathrm{pH} 2$ and extraction with ether, followed by vacuum distillation and drying with $\mathrm{MgSO}_{4}$, furnished a colourless oil, the half ethylester of methyl malonic acid $\left[\left(\mathrm{CO}_{2} \mathrm{H}\right) \mathrm{CHMeCO}_{2} \mathrm{Et}\right](24.8 \mathrm{~g}$, $0.17 \mathrm{~mol}, 84 \%)$. This latter product $(5.8 \mathrm{~g}, 0.040 \mathrm{~mol})$ was dissolved in $15 \mathrm{~mL}$ of dry ether and freshly distilled thionyl chloride was slowly added to it. Reflux for $2 \mathrm{~h}$ and elimination of the excess of $\mathrm{SOCl}_{2}$ and ether, under vacuum, furnished a clear yellow oil $\left[\left(\mathrm{CO}_{2} \mathrm{Et}\right) \mathrm{CHMeCOCl}\right]$, used without further purification in the following procedure. The production of the acid chloride was followed by TLC $\left(\mathrm{CH}_{2} \mathrm{Cl}_{2} / \mathrm{MeOH}\right.$ 9:1).

The acid chloride (5.6 g, $34 \mathrm{mmol}$ ) was dissolved in 15 $\mathrm{mL}$ of abs. ether. (S)-(+)-1-cyclohexylethylamine (CHA) (3.82 g, $30 \mathrm{mmol}$ ) or (R)-(+)-1-phenylethylamine (PEA) $(4.05 \mathrm{~g}, 33 \mathrm{mmol})$, together with triethylamine (TEA) (4.54 $\mathrm{g}, 6.24 \mathrm{~mL}, 45 \mathrm{mmol}$ ) in $10 \mathrm{~mL}$ of abs. ether were slowly added over the solution of the acid chloride, under $\mathrm{N}_{2}$, the temperature kept between $-25^{\circ} \mathrm{C}$ and $-30{ }^{\circ} \mathrm{C}$. The vigorous stirring of the two layers caused immediate precipitation of light yellow solids. The contact was kept for $1 \mathrm{~h}$, without cooling. Water $(20 \mathrm{~mL})$ was added with further stirring (15 min). The organic phase was treated with $\mathrm{H}_{2} \mathrm{SO}_{4} 5 \%$ (20mL) and $\mathrm{Na}_{2} \mathrm{CO}_{3} 5 \%(20 \mathrm{~mL})$. Solvent elimination and drying with $\mathrm{MgSO}_{4}$ furnished the amide-ester derivatives $\left[\left(\mathrm{CO}_{2} \mathrm{Et}\right) \mathrm{CHMeCONHR} *\right]$. Flash chromatography on silica gel column of the latter compounds (petroleum ether/acetone 4:1) furnished white amorphous powders, consisting of mixtures of the 2 diastereomers for both compounds. Their yields (1c: $3.59 \mathrm{~g}, 14.1 \mathrm{mmol}, 47 \%$; 1d: $3.61 \mathrm{~g}, 14.5 \mathrm{mmol}, 44 \%)$ were not optimised. The last compounds (1c, $1.28 \mathrm{~g}, 5 \mathrm{mmol}$ and $1 \mathrm{~d}, 1.24 \mathrm{~g}, 5 \mathrm{mmol})$ were stirred overnight with $\mathrm{KOH}(1.13 \mathrm{~g}, 20 \mathrm{mmol})$, dissolved in $25 \mathrm{~mL}$ of abs. ethanol. After solvent elimination, the residual solids were dissolved in water $(20 \mathrm{~mL})$, and extracted once with ether $(25 \mathrm{~mL})$. The aqueous phases were acidified with $\mathrm{HCl} 2 \mathrm{~N}(\mathrm{pH} 1)$ and extracted twice with ether $(20 \mathrm{~mL})$. Drying with $\mathrm{MgSO}_{4}$ and concentration furnished white amorphous solids. Crystallization from ether/petrol furnished the diastereomeric mixture of $\mathbf{1 a}$ (3.06 g, $13.5 \mathrm{mmol}$, total yield $45 \%)$ or $\mathbf{1 b}(2.99 \mathrm{~g}, 13.5$ mmol, total yield $41 \%$ ).

1a: N-[(1S)-Cyclohexylethyl)-2-methylmalonamic acid. White solid, m.p. $123-5^{\circ} \mathrm{C}$. IR (KBr), $\mathrm{cm}^{-1}: 3500$ (br, v O-H), 3300, 3100 (v N-H), 2950, 2900 (v C-H), 1725 (v $\mathrm{C}=\mathrm{O}), 1640(\mathrm{v} \mathrm{C}=\mathrm{ONH}$, amide $\mathrm{I}), 1560(\delta \mathrm{N}-\mathrm{H}, v \mathrm{C}-\mathrm{N}$, amide II). ${ }^{1} \mathrm{H}-\mathrm{NMR}\left(\mathrm{CDCl}_{3}, 300 \mathrm{MHz}, \delta\right): 0.86-1.03(1 \mathrm{H}$, $\mathrm{m}) ; 1.08\left[3 \mathrm{H}, \mathrm{d},{ }^{3} \mathrm{~J}=6.7 \mathrm{~Hz},-\mathrm{CH}(\mathrm{NH}) \mathrm{CH}_{3}\right], 1.12-1.38(5 \mathrm{H}$, $\mathrm{m}), 1.58-1.78(5 \mathrm{H}, \mathrm{m}), 1.45\left(3 \mathrm{H}, \mathrm{d},{ }^{3} \mathrm{~J}=7.1 \mathrm{~Hz},-\right.$ $\left.\mathrm{COCHCH}_{3}\right), 3.30\left(1 \mathrm{H}, \mathrm{q},{ }^{3} \mathrm{~J}=7.1 \mathrm{~Hz},-\mathrm{COCHCH}_{3}\right), 3.76-$ $3.83\left[1 \mathrm{H}, \mathrm{m},-\underline{\mathrm{CH}}(\mathrm{NH}) \mathrm{CH}_{3}\right], 6.86(1 \mathrm{H}, \mathrm{bs}, \mathrm{NH}), 11.61(1 \mathrm{H}$, bs, $\mathrm{OH})$ and ${ }^{13} \mathrm{C}-\mathrm{NMR}\left(\mathrm{CDCl}_{3}, 75 \mathrm{MHz}, \delta\right): 16.2 / 16.4$ $\left(\mathrm{CH}_{3}\right), 17.5\left(\mathrm{CH}_{3}\right), 26.0\left(\mathrm{CH}_{2}\right), 26.2\left(\mathrm{CH}_{2}\right), 28.9\left(\mathrm{CH}_{2}\right)$, $29.0\left(\mathrm{CH}_{2}\right), 29.0\left(\mathrm{CH}_{2}\right), 42.8(\mathrm{CH}), 45.2 / 45.3(\mathrm{CH}), 50.1$ $(\mathrm{d}, \underline{\mathrm{C}}-\mathrm{N}), 170.8 / 171.2(\mathrm{C}=\mathrm{O}), 174.4 / 174.5(\mathrm{C}=\mathrm{O})$. MS (70 $\mathrm{eV}$ ) (as methyl ester): $\mathrm{m} / \mathrm{z}(\%) 241(0.4)\left[\mathrm{M}^{+}\right], 226(0.2)$ $\left[\mathrm{M}^{+}-\mathrm{Me}\right], 158$ (34) $\left[\mathrm{M}^{+}-\mathrm{C}_{6} \mathrm{H}_{11}\right], 44$ (100). C,H-analysis $\mathrm{C}_{12} \mathrm{H}_{21} \mathrm{NO}_{3}$ (227.304), calculated: C63.41 H9.31 N6.16, experimental C63.85 H9.23 N6.38.

1b: N-[(1R)-1-Phenylethyl]-2-methylmalonamic acid. White needles, m.p. $102-104^{\circ} \mathrm{C}$. IR (KBr) cm ${ }^{-1}: 3400$ (br, v O-H), 3300 ( $v$ N-H), 3050 ( $v$ N-H), 1715 (v CO-O), 1620 ( $\vee \mathrm{C}=\mathrm{ONH}$, amide I), $1540(\delta \mathrm{N}-\mathrm{H}, v \mathrm{C}-\mathrm{N}$, amide II). ${ }^{1} \mathrm{H}-\mathrm{NMR}\left(\mathrm{CDCl}_{3}, 300 \mathrm{MHz}, \delta\right): 1.39\left(3 \mathrm{H}, \mathrm{d},{ }^{3} \mathrm{~J}=7.2 \mathrm{~Hz}\right.$, $\left.-\mathrm{COCHCH}_{3}\right), 1.46\left(3 \mathrm{H}, \mathrm{d},{ }^{3} \mathrm{~J}=6.7 \mathrm{~Hz},-\mathrm{CH}(\mathrm{NH}) \underline{\mathrm{CH}}_{3}\right), 3.34$ $\left(1 \mathrm{H}, \mathrm{q},{ }^{3} \mathrm{~J}=7.2 \mathrm{~Hz},-\mathrm{COCHCH}_{3}\right), 5.0-5.1(1 \mathrm{H}, \mathrm{m},-$ $\left.\underline{\mathrm{CH}}(\mathrm{NH}) \mathrm{CH}_{3}\right), 7.2-7.3(5 \mathrm{H}, \mathrm{m}, \mathrm{ArH}), 7.4-7.5(1 \mathrm{H}, \mathrm{m}, \mathrm{NH})$, $11.61(1 \mathrm{H}, \mathrm{s}, \mathrm{OH})$ and ${ }^{13} \mathrm{C}-\mathrm{NMR}\left(\mathrm{CDCl}_{3}, 75 \mathrm{MHz}, \delta\right): 16.2$ $\left(\mathrm{CH}_{3}\right), 22.1\left(\mathrm{CH}_{3}\right), 45.7(\mathrm{CH}), 49.8(\mathrm{CH}), 126.1(\mathrm{CH})$, $127.6(\mathrm{CH}), 128.8(\mathrm{CH}), 142.7(\mathrm{Cq}), 171.2(\mathrm{C}=\mathrm{O}), 174.8$ $(\mathrm{C}=\mathrm{O}) . \mathrm{MS}(70 \mathrm{eV}) \mathrm{m} / \mathrm{z}(\%)$ as methyl ester: $235(12)\left[\mathrm{M}^{+}\right]$, 
$220\left[\mathrm{M}^{+}-\mathrm{Me}\right], 120(100)\left[\mathrm{C}_{8} \mathrm{H}_{10} \mathrm{~N}^{+}\right], 106(91)\left[\mathrm{C}_{8} \mathrm{H}_{10}{ }^{+}\right], 105$ (95) $\left[\mathrm{C}_{8} \mathrm{H}_{9}{ }^{+}\right], 91$ (10), 88 (9), 79 (22), 77 (31), 59 (26). C,H-analysis: $\mathrm{C}_{12} \mathrm{H}_{15} \mathrm{O}_{3} \mathrm{~N}$ (221.256), calculated C65.14, H6.83, N6.33, experimental C64.44, H6.76, N6.36.

1c: N-[(1S)-1-Cyclohexylethyl)-2-methyl malonamic acid ethyl ester. White solid, m.p 123-126 ${ }^{\circ} \mathrm{C}$. IR (KBr) $\mathrm{cm}^{-1}$ : 3250 ( $\left.\mathrm{v} \mathrm{N}-\mathrm{H}\right), 3075$ ( $\left.\mathrm{v} \mathrm{N}-\mathrm{H}\right), 2950,2900,2850(\mathrm{v}$ $\mathrm{C}-\mathrm{H}), 1730$ ( $v \mathrm{CO}-\mathrm{O}$ ), 1620 ( $\vee \mathrm{C}=\mathrm{ONH}$, amide $\mathrm{I}), 1550$ ( $\delta$ $\mathrm{N}-\mathrm{H}, v \mathrm{C}-\mathrm{N}$, amide II), 1185 ( $v \mathrm{C}-\mathrm{O}-\mathrm{C}) .{ }^{13} \mathrm{C}-\mathrm{NMR}\left(\mathrm{CDCl}_{3}\right.$, $75 \mathrm{MHz}, \delta): 13.7\left(\mathrm{CH}_{3}\right), 14.7\left(\mathrm{CH}_{3}\right), 17.7\left(\mathrm{CH}_{3}\right), 26.0$ $\left(\mathrm{CH}_{2}\right), 26.2\left(\mathrm{CH}_{2}\right), 28.6\left(\mathrm{CH}_{2}\right), 28.9\left(\mathrm{CH}_{2}\right), 29.0\left(\mathrm{CH}_{2}\right)$, $43.0(\mathrm{CH}), 46.8(\mathrm{CH}), 49.3(\mathrm{CH}), 61.2\left(\mathrm{OCH}_{2}\right), 168.0$ $(\mathrm{C}=\mathrm{O}), 172.2(\mathrm{C}=\mathrm{O})$. MS (70 eV) m/z (\%): 210 (2) $\left[\mathrm{M}^{+}-\right.$ 45], 172 (42) [ $\left.\mathrm{M}^{+}-\mathrm{C}_{6} \mathrm{H}_{11}\right], 154$ (4) $\left[\mathrm{M}^{+}-\mathrm{C}_{5} \mathrm{H}_{9} \mathrm{O}_{2}\right], 146$ (10), $129(5)\left[\mathrm{C}_{6} \mathrm{H}_{9} \mathrm{O}_{3}{ }^{+}\right], 126(3)\left[\mathrm{M}^{+}-129\right], 111(3)\left[\mathrm{C}_{8} \mathrm{H}_{15}{ }^{+}\right], 55$ (15), 44. C,H-analysis: $\mathrm{C}_{14} \mathrm{H}_{25} \mathrm{O}_{3} \mathrm{~N}$ (255.183), calculated C65.85, H9.87, N5.49, experimental C66.18, H10.19, N5.52.

1d: $\mathrm{N}$-[(1R)-1-Phenylethyl]-2-methyl malonamic acid ethyl ester. Amorphous white solid, m.p $65-66^{\circ} \mathrm{C}$. IR (KBr) $\mathrm{cm}^{-1}: 3450,3400$ (br, v O-H), 3250 ( $\left.\mathrm{v} \mathrm{N}-\mathrm{H}\right), 3050$ (v N-H), 1730 ( $v$ CO-O), 1620 ( $v \mathrm{C}=\mathrm{ONH}$, amide I), $1540(\delta \mathrm{N}-\mathrm{H}$, v C-N, amide II), 1185 (v C-O-C). ${ }^{1} \mathrm{H}-\mathrm{NMR}\left(\mathrm{CDCl}_{3}, 300\right.$ $\mathrm{MHz}, \delta)$ : major diastereomer: $1.21(3 \mathrm{H}, \mathrm{t}, \mathrm{J}=7.1 \mathrm{~Hz}$, $\left.-\mathrm{OCH}_{2} \underline{\mathrm{CH}}_{3}\right), 1.40\left(3 \mathrm{H}, \mathrm{d}, \mathrm{J}=7.2 \mathrm{~Hz},-\mathrm{COCHCH}_{3}\right), 1.47$ $\left(3 \mathrm{H}, \mathrm{d}, \mathrm{J}=7.0 \mathrm{~Hz},-\mathrm{CH}(\mathrm{NH}) \underline{\mathrm{CH}}_{3}\right), 3.28(1 \mathrm{H}, \mathrm{d}, \mathrm{J}=7.2 \mathrm{~Hz}$, $\left.-\mathrm{COCHCH}_{3}\right), 4.14\left(2 \mathrm{H}, \mathrm{q}, \mathrm{J}=7.1 \mathrm{~Hz},-\mathrm{OCH}_{2} \mathrm{CH}_{3}\right), 5.0-5.1$ $\left(1 \mathrm{H}, \mathrm{m},-\mathrm{CH}(\mathrm{NH}) \mathrm{CH}_{3}\right), 6.9(1 \mathrm{H}, \mathrm{bs}, \mathrm{NH}), 7.2-7.4(5 \mathrm{H}, \mathrm{m}$, $\mathrm{ArH})$; minor diastereomer: similar signals, except 1.27 $\left(3 \mathrm{H}, \mathrm{t}, \mathrm{J}=7.1 \mathrm{~Hz},-\mathrm{OCH}_{2} \underline{\mathrm{CH}}_{3}\right), 1.39(3 \mathrm{H}, \mathrm{d}, \mathrm{J}=7.2 \mathrm{~Hz}$, $\left.-\mathrm{COCHCH}_{3}\right)$ and $3.26\left(1 \mathrm{H}, \mathrm{d}, \mathrm{J}=7.2 \mathrm{~Hz},-\mathrm{COCHCH}_{3}\right)$. ${ }^{13} \mathrm{C}-\mathrm{NMR}\left(\mathrm{CDCl}_{3}, 75 \mathrm{MHz}, \delta\right) 14.1\left(\mathrm{CH}_{3}\right), 14.8\left(\mathrm{CH}_{3}\right), 21.8$ $\left(\mathrm{CH}_{3}\right), 46.8(\mathrm{CH}), 48.8(\mathrm{CH}), 61.5\left(\mathrm{OCH}_{2}\right), 125.9(\mathrm{CH})$, $126.0(\mathrm{CH}), 127.2(\mathrm{CH}), 128.4(\mathrm{CH}), 128.5(\mathrm{CH}), 143.1$ (Cq), $168.0(\mathrm{C}=\mathrm{O}), 172.1(\mathrm{C}=\mathrm{O})$. $\mathrm{MS}(70 \mathrm{eV}) \mathrm{m} / \mathrm{z}(\%): 250$ (1) $\left[\mathrm{M}^{+}+1\right], 249(1)\left[\mathrm{M}^{+}\right], 234(3)\left[\mathrm{M}^{+}-\mathrm{Me}\right], 120$ (100) $\left[\mathrm{C}_{8} \mathrm{H}_{10} \mathrm{~N}^{+}\right], 105$ (40) $\left[\mathrm{C}_{8} \mathrm{H}_{9}^{+}\right], 77$ (13). C,H-analysis: $\mathrm{C}_{14} \mathrm{H}_{19} \mathrm{O}_{3} \mathrm{~N}$ (249.309), calculated C67.45, H7.68, N5.62, experimental C66.84, H7.76, N5.64.

Synthesis of co-acids

2f: Diethylphosphonoacetic acid. Triethylphosphonoacetate $(22.42 \mathrm{~g}, 0.1 \mathrm{~mol})$ in $100 \mathrm{~mL}$ of abs. EtOH was added to a solution of $\mathrm{KOH}(5.6 \mathrm{~g}, 0.1 \mathrm{~mol})$ in $100 \mathrm{~mL}$ of abs. EtOH and stirred for $24 \mathrm{~h}$. TLC (ethyl acetate/ $\mathrm{MeOH} 2: 1$ ) was used to follow the reaction. After solvent elimination, the residual oil was dissolved in water (20 $\mathrm{mL})$, and extracted once with ether $(25 \mathrm{~mL})$, leading to the recovery of triethylphosphonoacetate $(2.98 \mathrm{~g}, 0.012 \mathrm{~mol}$, $12 \%)$. The aqueous phase was acidified with $\mathrm{HCl} 2 \mathrm{~N}(\mathrm{pH}$ 1) and extracted twice with ether $(20 \mathrm{~mL})$. Drying with $\mathrm{MgSO}_{4}$ and concentration furnished $\mathbf{2 \mathbf { f } ^ { 1 7 }}$, a light yellow oil (11.0 g, 0.056 mol, 56\%). ${ }^{1} \mathrm{H}-\mathrm{NMR}$ (300 MHz, $\delta$ ): 1.13 (6
$\left.\mathrm{H}, \mathrm{t}, \mathrm{J}=7.5 \mathrm{~Hz},-\mathrm{OCH}_{2} \mathrm{CH}_{3}\right), 2.80\left(2 \mathrm{H}, \mathrm{d}, \mathrm{J}_{\mathrm{H}-\mathrm{P}}=22.5 \mathrm{~Hz}\right.$, $\left.-\underline{\mathrm{CH}}_{2} \mathrm{P}-\right)$, 3.9-4.1 (4H, m, $\left.-\underline{\mathrm{OCH}}_{2} \mathrm{CH}_{3}\right), 10.5(1 \mathrm{H}, \mathrm{bs}, \mathrm{OH})$. ${ }^{13} \mathrm{C}-\mathrm{NMR}\left(\mathrm{CDCl}_{3}, 75 \mathrm{MHz}, \delta\right): 16.05\left(\mathrm{CH}_{3}\right), 16.13\left(\mathrm{CH}_{3}\right)$, $33.06\left(\mathrm{CH}_{2}\right), 34.85\left(\mathrm{CH}_{2}\right), 63.39\left(\mathrm{CH}_{2}\right), 167.64(\mathrm{Cq})$.

\section{Kolbe electrolysis}

The following description is typical for Kolbe electrolysis and methods for work-up and isolation of products. After partial neutralisation (methanolic $\mathrm{KOH}, 5$ to $10 \%$ ), 1a-b $(0.5$ to $1 \mathrm{mmol})$ were submitted to Kolbe oxidations [undivided jacketed cell, $\mathrm{Pt}$ (anode, cathode), $\mathrm{MeOH}, 200$ to $250 \mathrm{~mA} / \mathrm{cm}^{2}, 1.3-1.5 \mathrm{~F} \mathrm{~mol}^{-1}$, co-acid in excess $\left.(10 \mathrm{x})\right]$, with a temperature range between 10 and $60^{\circ} \mathrm{C}$. To get this temperature inside the cell, it is necessary to use a cooling device and the cryostat was cooled earlier to $-40{ }^{\circ} \mathrm{C}$. The electrolyses were always carried out with the diastereomeric mixture of the carboxylic acids $\mathbf{1 a}$ or $\mathbf{1 b}$. The end of the reaction was monitored by $\mathrm{pH}$ measurement (acid to neutral). When passivation or coverage of electrodes were noticed, the technique of polarity inversion was used. Adequate work up and fractionation, through flash chromatography, furnished products from Kolbe (K) and non-Kolbe $(\mathrm{nK})$ pathways. The yields in Table 1 are related to the main acid (1a, $\mathbf{1 b}$ or $\mathbf{2 e}$ ) present. Homocoupling of the co-acids was not quantified. The absolute configurations of the purified reaction products were not determined. Relevant data from the main products of Kolbe electrolyses (Table 1, Fig. 2), mainly mixed Kolbe dimers (MKD), are included.

3a, $\mathbf{R}=\mathbf{C}_{5} \mathrm{H}_{11}: \mathrm{N}-[(1 \mathrm{~S})-1-\mathrm{Cyclohexylethyl}]-2-$ methylheptanamide. White solid, m.p. 92-5 ${ }^{\circ} \mathrm{C}$. ${ }^{1} \mathrm{H}-\mathrm{NMR}(300$ $\mathrm{MHz}, \delta): 0.87\left(3 \mathrm{H}, \mathrm{t},{ }^{3} \mathrm{~J}=6.5 \mathrm{~Hz},-\mathrm{CH}_{2} \mathrm{CH}_{3}\right), 0.8-1.15(5 \mathrm{H}$, $\left.\mathrm{m},-\underline{\mathrm{CHCH}}_{2}^{-}\right), 1.07\left[3 \mathrm{H}, \mathrm{d},{ }^{3} \mathrm{~J}=6.7 \mathrm{~Hz},-\mathrm{CH}(\mathrm{NH}) \underline{\mathrm{CH}}_{3}\right], 1.10$ $\left(3 \mathrm{H}, \mathrm{d},{ }^{3} \mathrm{~J}=6.7 \mathrm{~Hz}, \mathrm{OCHCH}_{3}\right), 1.18-1.42\left(8 \mathrm{H}, \mathrm{m},-\underline{\mathrm{CH}}_{2}^{-}\right)$, 1.55-1.79 (6H, m, $\left.-\underline{\mathrm{CH}}_{2}-\right), 2.07-2.17\left(1 \mathrm{H}, \mathrm{m}, \mathrm{COCHCH}_{3}\right)$, 3.77-3.89 (1H, m, $\left.-\underline{\mathrm{CH}}(\mathrm{NH}) \mathrm{CH}_{3}\right), 5.27(1 \mathrm{H}, \mathrm{bs},-\mathrm{NH})$. ${ }^{13} \mathrm{C}-\mathrm{NMR}\left(\mathrm{CDCl}_{3}, 75 \mathrm{MHz}, \delta\right): 14.0\left(\mathrm{CH}_{3}\right), 18.1\left(\mathrm{CH}_{3}\right)$, $18.1\left(\mathrm{CH}_{3}\right), 22.5\left(\mathrm{CH}_{2}\right), 26.1\left(\mathrm{CH}_{2}\right), 26.4\left(\mathrm{CH}_{2}\right), 27.1$ $\left(\mathrm{CH}_{2}\right), 27.1\left(\mathrm{CH}_{2}\right), 29.0\left(\mathrm{CH}_{2}\right), 29.1\left(\mathrm{CH}_{2}\right), 31.8\left(\mathrm{CH}_{2}\right)$, $34.4\left(\mathrm{CH}_{2}\right), 41.9(\mathrm{CH}), 43.2(\mathrm{CH}), 49.2(\mathrm{CH}), 175.7(\mathrm{C}=\mathrm{O})$. MS (70 eV) m/z (\%): 253 (2) $\left[\mathrm{M}^{+}\right], 183(16)\left[\mathrm{M}^{+}-\mathrm{C}_{5} \mathrm{H}_{10}\right]$, 171 (6) $\left[\mathrm{M}^{+}-\mathrm{C}_{6} \mathrm{H}_{10}\right], 170$ (39) $\left[\mathrm{M}^{+}-\mathrm{C}_{6} \mathrm{H}_{11}\right], 144$ (34) $\left[\mathrm{M}^{+}-\right.$ $\left.\mathrm{C}_{8} \mathrm{H}_{13}\right], 127$ (6), 44 (100). C,H-analysis $\mathrm{C}_{16} \mathrm{H}_{31} \mathrm{ON}$ (253.2406), calculated C75.82 H12.34 N5.53, experimental C75.88 H12.35 N5.48. The diastereomers were separated, but the NMR data for the pure compounds were not obtained.

3a, $\mathbf{R}=\mathbf{C H}_{2} \mathrm{SiMe}_{3}: \mathrm{N}-[(1 \mathrm{~S})-1-$ Cyclohexylethyl]-2methyl-3-trimethylsilyl propanamide. White solid, m.p. 62-64 ${ }^{\circ} \mathrm{C} .{ }^{1} \mathrm{H}-\mathrm{NMR}(300 \mathrm{MHz}, \delta): 0.05\left[9 \mathrm{H}, \mathrm{s},-\mathrm{Si}\left(\underline{\mathrm{CH}}_{3}\right) 3\right]$, $0.65(1 \mathrm{H}, \mathrm{dd}, \mathrm{J}=15.0$ and $8.3 \mathrm{~Hz},-\mathrm{SiCH} \underline{\mathrm{H}}-), 0.93(1 \mathrm{H}$, dd, $\mathrm{J}=15.0$ and $6.0 \mathrm{~Hz},-\mathrm{SiC} \underline{\mathrm{H}} \mathrm{H}-), 0.87-1.37\left(6 \mathrm{H}, \mathrm{m},-\underline{\mathrm{CH}}_{2}-\right)$, $1.04\left(3 \mathrm{H}, \mathrm{d},{ }^{3} \mathrm{~J}=6.8 \mathrm{~Hz}, \mathrm{CHCH}_{3}\right), 1.13\left(3 \mathrm{H}, \mathrm{d},{ }^{3} \mathrm{~J}=7.0 \mathrm{~Hz}\right.$, 
$\left.\mathrm{CHCH}_{3}\right), 1.5-1.8(5 \mathrm{H}, \mathrm{m}), 2.26(1 \mathrm{H}, \mathrm{m},-\mathrm{COCHCH}), 3.72-$ $3.86\left[1 \mathrm{H}, \mathrm{m},-\underline{\mathrm{CH}}(\mathrm{NH}) \mathrm{CH}_{3}\right], 5.20(1 \mathrm{H}, \mathrm{bs},-\mathrm{NH}) .{ }^{13} \mathrm{C}-\mathrm{NMR}$ $(75 \mathrm{MHz}, \delta):-1.0\left(3 \mathrm{x} \mathrm{CH}_{3}\right), 17.8\left(\mathrm{CH}_{3}\right), 21.2\left(\mathrm{CH}_{3}\right), 21.9$ $\left(\mathrm{CH}_{2}\right), 26.1\left(\mathrm{CH}_{2}\right), 26.4\left(\mathrm{CH}_{2}\right), 28.9\left(\mathrm{CH}_{2}\right), 29.0\left(\mathrm{CH}_{2}\right)$, $29.1\left(\mathrm{CH}_{2}\right), 37.9(\mathrm{CH}), 43.0(\mathrm{CH}), 48.9(\mathrm{CH}), 176.6(\mathrm{C}=\mathrm{O})$. MS (70 eV) m/z (\%): $342(10)\left[\mathrm{M}^{+}+\mathrm{SiMe}_{3}\right], 270(5)\left[\mathrm{M}^{+}\right.$ + 1], 254 (68) [M+ - Me], 226 (4), 196 (8), 178 (11), 160 (30), 144 (100), 73 (93), 44 (98). C,H-analysis $\mathrm{C}_{15} \mathrm{H}_{31} \mathrm{ONSi}$ (269.217), calculated C66.86 H11.60 N5.20, experimental C66.92 H11.64 N5.20.

3b, $\mathbf{R}=\mathbf{C}_{5} \mathrm{H}_{11}: \mathrm{N}$-[(1R)-1-Phenylethyl]-2-methylheptanamide. White amorphous powder, m.p. $88-90{ }^{\circ} \mathrm{C}$.

The diastereomeric mixture was separated and ${ }^{1} \mathrm{H}$ and ${ }^{13} \mathrm{C}$-NMR obtained for the pure compounds:

3b' (less polar): ${ }^{1} \mathrm{H}-\mathrm{NMR}\left(300 \mathrm{MHz}, \mathrm{CDCl}_{3}, \delta\right): 0.88$ $\left(3 \mathrm{H}, \mathrm{t},{ }^{3} \mathrm{~J}=7.0 \mathrm{~Hz},-\mathrm{CH}_{2} \mathrm{CH}_{3}\right), 1.11\left(3 \mathrm{H}, \mathrm{d},{ }^{3} \mathrm{~J}=7.0 \mathrm{~Hz}\right.$, $\left.-\mathrm{COCHCH} 3{ }_{3}\right), 1.27-1.41\left[6 \mathrm{H}, \mathrm{m},-\left(\underline{\mathrm{CH}}_{2}\right)_{3}\right], 1.49\left(3 \mathrm{H}, \mathrm{d},{ }^{3} \mathrm{~J}=\right.$ $\left.7.0 \mathrm{~Hz},-\mathrm{CH}(\mathrm{NH}) \mathrm{CH}_{3}\right), 1.6-1.7\left(2 \mathrm{H}, \mathrm{m},-\mathrm{CH}_{2}-\right), 2.08-2.20$ $(1 \mathrm{H}, \mathrm{m},-\mathrm{COCHCH})_{3}, 5.1-5.2\left(1 \mathrm{H}, \mathrm{m}, \underline{\mathrm{CH}}(\mathrm{NH}) \mathrm{CH}_{3}\right), 5.61-$ $5.63(1 \mathrm{H}, \mathrm{bs},-\mathrm{NH}), 7.23-7.36(5 \mathrm{H}, \mathrm{m}, \mathrm{ArH}) .{ }^{13} \mathrm{C}-\mathrm{NMR}(75$ $\left.\mathrm{MHz}, \mathrm{CDCl}_{3}, \delta\right): 14.2\left(\mathrm{CH}_{3}\right), 18.1\left(\mathrm{CH}_{3}\right), 21.9\left(\mathrm{CH}_{3}\right), 22.8$ $\left(\mathrm{CH}_{2}\right), 27.4\left(\mathrm{CH}_{2}\right), 32.0\left(\mathrm{CH}_{2}\right), 34.6\left(\mathrm{CH}_{2}\right), 41.9(\mathrm{CH}), 48.6$ $(\mathrm{CH}), 126.4$ ( $2 \times \mathrm{CH}$ ar. $), 127.5$ ( $\mathrm{CH}$ ar. $), 128.9(2 \times \mathrm{CH}$ ar.), $143.6(\mathrm{Cq}), 175.8(\mathrm{C}=\mathrm{O})$. C,H-analysis $\mathrm{C}_{16} \mathrm{H}_{25} \mathrm{ON}$ (247.1936), calculated C77.67 H10.19 N5.66, experimental C77.38 H10.15 N5.76.

3b"' (more polar): ${ }^{1} \mathrm{H}-\mathrm{NMR}\left(300 \mathrm{MHz}, \mathrm{CDCl}_{3}, \delta\right): 0.84$ $\left(3 \mathrm{H}, \mathrm{t},{ }^{3} \mathrm{~J}=7.0 \mathrm{~Hz}\right), 1.14\left(3 \mathrm{H}, \mathrm{d},{ }^{3} \mathrm{~J}=6.8 \mathrm{~Hz}\right), 1.2-1.3(6 \mathrm{H}$, $\mathrm{m}), 1.48\left(3 \mathrm{H}, \mathrm{d},{ }^{3} \mathrm{~J}=6.8 \mathrm{~Hz}\right), 1.6-1.7(2 \mathrm{H}, \mathrm{m}), 2.1-2.2(1 \mathrm{H}$, m), 5.1-5.2 $(1 \mathrm{H}, \mathrm{m}), 5.61-5.64(1 \mathrm{H}, \mathrm{bs}, \mathrm{NH}), 7.23-7.36(5 \mathrm{H}$, $\mathrm{m})$. The assignment of the signals is the same as in $\mathbf{3 b}$ '. ${ }^{13} \mathrm{C}$-NMR $\left(75 \mathrm{MHz}, \mathrm{CDCl}_{3}, \delta\right): 14.0\left(\mathrm{CH}_{3}\right), 18.1\left(\mathrm{CH}_{3}\right)$, $21.8\left(\mathrm{CH}_{3}\right), 22.7\left(\mathrm{CH}_{2}\right), 27.3\left(\mathrm{CH}_{2}\right), 32.0\left(\mathrm{CH}_{2}\right), 34.6$ $\left(\mathrm{CH}_{2}\right), 41.9(\mathrm{CH}), 48.6(\mathrm{CH}), 126.3(\mathrm{CH}$ ar. $), 126.4((\mathrm{CH}$ ar.), 127.5 (CH ar.), 128.8 ( $\mathrm{CH}$ ar.), 129.0 (CH ar.), 143.7 $(\mathrm{Cq}), 175.8(\mathrm{C}=\mathrm{O})$; MS (70 eV) m/z (\%): 247 (29) $\left[\mathrm{M}^{+}\right]$, $190(8)\left[\mathrm{M}^{+}-57\right], 177(63), 120(18)\left[\mathrm{C}_{8} \mathrm{H}_{10} \mathrm{~N}^{+}\right], 106(51)$, 105 (100) $\left[\mathrm{C}_{8} \mathrm{H}_{9}{ }^{+}\right], 104$ (35), 77 (16), 57 (36).

3b, $\mathbf{R}=\mathbf{C H}_{2} \mathrm{PO}\left(\mathrm{OC}_{2} \mathrm{H}_{5}\right)_{2}: \mathrm{N}-[(1 \mathrm{R})-1$-Phenylethyl]-2methyl-3-diethylphosphonylpropanamide. Evidenced only by GC/MS. MS (70 eV) m/z (\%): $328(3)\left[\mathrm{M}^{+}+1\right], 312$ (2) $\left[\mathrm{M}^{+-} 15\right], 207(23)\left[\mathrm{M}^{+}-120\right], 120(100)\left[\mathrm{C}_{8} \mathrm{H}_{10} \mathrm{~N}^{+}\right]$.

4a: N-[(1S)-1-Cyclohexylethyl]-2-methoxypropanamide. ${ }^{1} \mathrm{H}-\mathrm{NMR}\left(300 \mathrm{MHz}, \mathrm{CDCl}_{3}, \delta\right)$ : 0.87-1.37 (5H, m), $1.04\left(3 \mathrm{H}, \mathrm{d},{ }^{3} \mathrm{~J}=6.8 \mathrm{~Hz}, \mathrm{CHNHCH}{ }_{3}\right), 1.27\left(3 \mathrm{H}, \mathrm{d},{ }^{3} \mathrm{~J}=7.0\right.$ $\left.\mathrm{Hz},-\mathrm{COCHCH}_{3}\right), 1.52-1.72\left(6 \mathrm{H}, \mathrm{m},-\mathrm{CH}_{2}-\right), 3.33(3 \mathrm{H}, \mathrm{s}$, $\mathrm{OMe}), 3.62\left(1 \mathrm{H}, \mathrm{q}, \mathrm{J}=7.0 \mathrm{~Hz}, \mathrm{COCHCH}_{3}\right), 3.71-3.82(1 \mathrm{H}$,

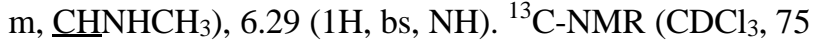
$\mathrm{MHz}, \delta): 17.9\left(\mathrm{CH}_{3}\right), 18.1\left(\mathrm{CH}_{3}\right), 26.2\left(\mathrm{CH}_{2}\right), 26.4\left(\mathrm{CH}_{2}\right)$, $28.9\left(\mathrm{CH}_{2}\right), 29.0\left(\mathrm{CH}_{2}\right), 29.2\left(\mathrm{CH}_{2}\right), 43.2(\mathrm{CH}), 48.6(\mathrm{CH})$, $57.6\left(\mathrm{CH}_{3}\right), 78.4(\mathrm{CH}), 172.3(\mathrm{C}=\mathrm{O})$. MS $(70 \mathrm{eV}) \mathrm{m} / \mathrm{z}(\%)$ : $214(8)\left[\mathrm{M}^{+}+1\right], 213(1)\left[\mathrm{M}^{+}\right], 183(4)\left[\mathrm{M}^{+}-\mathrm{CH}_{2} \mathrm{O}\right], 130$ (40), 104 (32), 69 (18), 59 (81), 44 (100). 5a: N-[(1S)-1-Cyclohexylethyl]-acrylamide. White solid, m.p. $70-72{ }^{\circ} \mathrm{C} .{ }^{1} \mathrm{H}-\mathrm{NMR}\left(300 \mathrm{MHz}, \mathrm{CDCl}_{3}, \delta\right)$ : 0.8-1.4 (5H, m), $1.13\left(3 \mathrm{H}, \mathrm{d},{ }^{3} \mathrm{~J}=6.8 \mathrm{~Hz}, \mathrm{CHNHCH}_{3}\right)$, 1.64-1.82 (6H, m), 3.9-4.2 (1H, m, $\left.-\underline{-} \mathrm{HNHCH}_{3}\right), 5.58(1 \mathrm{H}$, $\left.\mathrm{dd},{ }^{3} \mathrm{~J}=10.2 \mathrm{~Hz},{ }^{3} \mathrm{~J}=1.5 \mathrm{~Hz},-\underline{\mathrm{CH}}=\mathrm{CH}_{2}\right), 5.67(1 \mathrm{H}, \mathrm{bs}$, $\mathrm{NH}), 6.11\left(1 \mathrm{H}, \mathrm{dd}, \mathrm{J}_{\mathrm{gem}}=17.0 \mathrm{~Hz},{ }^{3} \mathrm{~J}=10.2 \mathrm{~Hz}, \mathrm{CH}=\mathrm{CH}_{2}\right)$, $6.23\left(1 \mathrm{H}, \mathrm{dd}, \mathrm{J}_{\mathrm{gem}}=17.0 \mathrm{~Hz},{ }^{3} \mathrm{~J}=10.2 \mathrm{~Hz}, \mathrm{CH}=\mathrm{CH}_{2}\right)$. ${ }^{13} \mathrm{C}$-NMR $\left(\mathrm{CDCl}_{3}, 75 \mathrm{MHz}, \delta\right): 17.8\left(\mathrm{CH}_{3}\right), 26.1\left(\mathrm{CH}_{2}\right)$, $26.3\left(\mathrm{CH}_{2}\right), 28.8\left(\mathrm{CH}_{2}\right), 29.0\left(\mathrm{CH}_{2}\right), 29.1\left(\mathrm{CH}_{2}\right), 43.1(\mathrm{CH})$, $49.4(\mathrm{CH}), 125.8\left(\mathrm{CH}_{2}\right), 131.3(\mathrm{CH}), 164.8(\mathrm{C}=\mathrm{O})$. MS $(70$ eV) m/z (\%): $182(36)\left[\mathrm{M}^{+}+1\right], 181(5)\left[\mathrm{M}^{+}\right], 166(5)\left[\mathrm{M}^{+}-\right.$ $\mathrm{Me}$, 98 (100) $\left[\mathrm{M}^{+}-\mathrm{C}_{6} \mathrm{H}_{11}\right], 84$ (8) $\left[\mathrm{C}_{6} \mathrm{H}_{12}{ }^{+}\right], 72$ (23), 55 (81), 44 (91).

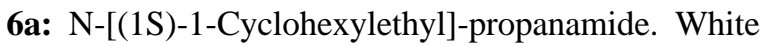
solid, m.p. 107-110 ${ }^{\circ} \mathrm{C} .{ }^{1} \mathrm{H}-\mathrm{NMR}\left(300 \mathrm{MHz}, \mathrm{CDCl}_{3}, \delta\right)$ : 0.80-1.35 (5H, m), $1.09\left(3 \mathrm{H}, \mathrm{d}, \mathrm{J}=6.8 \mathrm{~Hz},-\mathrm{CHNHCH}_{3}\right)$, $1.27\left(3 \mathrm{H}, \mathrm{J}=7.5 \mathrm{~Hz},-\mathrm{CH}_{2} \mathrm{CH}_{3}\right), 1.54-1.78(6 \mathrm{H}, \mathrm{m}), 2.12$ $\left(2 \mathrm{H}, \mathrm{q}, \mathrm{J}=7.5 \mathrm{~Hz},-\mathrm{CH}_{2} \mathrm{CH}_{3}\right), 3.75-3.90(1 \mathrm{H}, \mathrm{m}$,

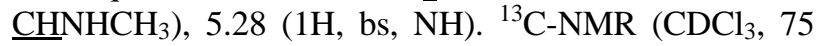
$\mathrm{MHz}, \delta): 10.0\left(\mathrm{CH}_{3}\right), 17.8\left(\mathrm{CH}_{3}\right), 26.2\left(\mathrm{CH}_{2}\right), 26.3\left(\mathrm{CH}_{2}\right)$, $26.4\left(\mathrm{CH}_{2}\right), 29.0\left(\mathrm{CH}_{2}\right), 29.2\left(\mathrm{CH}_{2}\right), 30.0\left(\mathrm{CH}_{2}\right), 43.1(\mathrm{CH})$, $49.1(\mathrm{CH}), 173.0(\mathrm{C}=\mathrm{O})$. MS (70 eV) m/z (\%): $184(10)$ $\left[\mathrm{M}^{+}+1\right], 154$ (2) $\left[\mathrm{M}^{+}-\mathrm{C}_{2} \mathrm{H}_{5}\right], 100$ (16), 74 (13), 44 (100).

4b: N-[(1R)-1-Phenylethyl]-2-methoxypropanamide. The presence of two diastereomers was evidenced. They have not been separated. ${ }^{1} \mathrm{H}-\mathrm{NMR}\left(300 \mathrm{MHz}, \mathrm{CDCl}_{3}, \delta\right)$ : major diastereomer: $1.41(3 \mathrm{H}, \mathrm{d}, \mathrm{J}=7 \mathrm{~Hz}), 1.50(3 \mathrm{H}, \mathrm{d}, \mathrm{J}$ $=6.7 \mathrm{~Hz}), 3.37(3 \mathrm{H}, \mathrm{s}, \mathrm{OMe}), 3.75(1 \mathrm{H}, \mathrm{q}, \mathrm{J}=7.0), 5.1-5.2$ $(1 \mathrm{H}, \mathrm{m}), 6.7-6.9(1 \mathrm{H}, \mathrm{bs}, \mathrm{NH}), 7.3-7.4(5 \mathrm{H}, \mathrm{m}, \mathrm{ArH})$; minor diastereomer: similar signals, except $\delta 1.37(3 \mathrm{H}, \mathrm{d}, \mathrm{J}=7$ $\mathrm{Hz}), 1.51(3 \mathrm{H}, \mathrm{d}, \mathrm{J}=6.7 \mathrm{~Hz}), 3.41(3 \mathrm{H}, \mathrm{s})$ and $3.80(1 \mathrm{H}, \mathrm{q}$, $\mathrm{J}=7 \mathrm{~Hz})$. MS (70 eV) m/z (\%): $208(4)\left[\mathrm{M}^{+}+1\right], 207$ (14) $\left[\mathrm{M}^{+}\right], 177(10)\left[\mathrm{M}^{+}-\mathrm{CH}_{2} \mathrm{O}\right], 175$ (29), $105(49)\left[\mathrm{C}_{8} \mathrm{H}_{9}^{+}\right]$.

5b: $\mathrm{N}-\left[(1 \mathrm{R})-1-\mathrm{Phenylethyl]}\right.$-acrylamide. ${ }^{1} \mathrm{H}-\mathrm{NMR}$ $\left(300 \mathrm{MHz}, \mathrm{CDCl}_{3}, \delta\right): 1.52(3 \mathrm{H}, \mathrm{d}, \mathrm{J}=6.8 \mathrm{~Hz}$, $\left.\mathrm{CH}(\mathrm{NH}) \mathrm{CH}_{3}\right), 5.1-5.3\left(1 \mathrm{H}, \mathrm{m}, \mathrm{CH}(\mathrm{NH}) \mathrm{CH}_{3}\right), 5.62(1 \mathrm{H}, \mathrm{dd}$, $\left.\mathrm{J}_{\mathrm{gem}}=10.2 \mathrm{~Hz},{ }^{3} \mathrm{~J}=1.5 \mathrm{~Hz},-\mathrm{CH}=\mathrm{CH}_{2}\right), 5.91(1 \mathrm{H}, \mathrm{bs}, \mathrm{NH})$, $6.08\left(1 \mathrm{H}, \mathrm{dd}, \mathrm{J}_{\mathrm{gem}}=17.0 \mathrm{~Hz},{ }^{3} \mathrm{~J}=10.2 \mathrm{~Hz}, \mathrm{CH}=\mathrm{CH}_{2}\right), 6.28$ $\left(1 \mathrm{H}, \mathrm{dd}, \mathrm{J}_{\mathrm{gem}}=17.0 \mathrm{~Hz},{ }^{3} \mathrm{~J}=1.5 \mathrm{~Hz}, \mathrm{CH}=\mathrm{CH}_{2}\right), 7.2-7.3(5 \mathrm{H}$, m, ArH). ${ }^{13} \mathrm{C}$-NMR $(75 \mathrm{MHz}, \delta): 21.8\left(\mathrm{CH}_{3}\right), 49.1(\mathrm{CH})$, $126.5(2 \times \mathrm{CH}), 126.7\left(\mathrm{CH}_{2}\right), 127.7(\mathrm{CH}), 128.9(2 \times \mathrm{CH})$, $131.4(\mathrm{CH}), 143.6(\mathrm{Cq}), 172.3(\mathrm{C}=\mathrm{O})$. MS $(70 \mathrm{eV}) \mathrm{m} / \mathrm{z}(\%)$ : 175 (30) $\left[\mathrm{M}^{+}\right], 160$ (39) $\left[\mathrm{M}^{+}-\mathrm{Me}\right], 131(54)\left[\mathrm{M}^{+}-44\right], 120$ (30) $\left[\mathrm{C}_{8} \mathrm{H}_{10} \mathrm{~N}^{+}\right], 106$ (100), 104 (32), 79 (17), 77 (27), 55 (55).

6b: $\mathrm{N}-\left[(1 \mathrm{R})-1-\mathrm{Phenylethyl]}\right.$-propanamide. ${ }^{1} \mathrm{H}-\mathrm{NMR}$ (300 MHz, $\left.\mathrm{CDCl}_{3}, \delta\right): 1.20\left(3 \mathrm{H}, \mathrm{t}, \mathrm{J}=7.0 \mathrm{~Hz}, \mathrm{CH}_{2} \mathrm{CH}_{3}\right)$, $1.50\left(3 \mathrm{H}, \mathrm{d}, \mathrm{J}=6.8 \mathrm{~Hz}, \mathrm{CH}(\mathrm{NH}) \underline{\mathrm{CH}}_{3}\right), 2.23(2 \mathrm{H}, \mathrm{q}, \mathrm{J}=7.0$ $\left.\mathrm{Hz}, \mathrm{CH}_{2} \mathrm{CH}_{3}\right), 5.1-5.3\left(1 \mathrm{H}, \mathrm{m}, \underline{\mathrm{CH}}(\mathrm{NH}) \mathrm{CH}_{3}\right), 5.67(1 \mathrm{H}, \mathrm{bs}$, $\mathrm{NH}), 7.3-7.4(5 \mathrm{H}, \mathrm{m}, \mathrm{ArH}) .{ }^{13} \mathrm{C}-\mathrm{NMR}(75 \mathrm{MHz}, \delta): 10.0$ $\left(\mathrm{CH}_{3}\right), 21.9\left(\mathrm{CH}_{3}\right), 30.0\left(\mathrm{CH}_{2}\right), 48.8(\mathrm{CH}), 126.4(2 \times \mathrm{CH})$, 127.6 (CH), $128.9(2 \times \mathrm{CH}), 143.5(\mathrm{Cq}), 172.5(\mathrm{C}=\mathrm{O}) . \mathrm{MS}$ $(70 \mathrm{eV}) \mathrm{m} / \mathrm{z}(\%): 178(7)\left[\mathrm{M}^{+}+1\right], 177(63)\left[\mathrm{M}^{+}\right], 162(18)$ 
[M+-Me], $120(44)\left[\mathrm{C}_{8} \mathrm{H}_{10} \mathrm{~N}^{+}\right], 106$ (100), 105 (50), 104 (42).

8a: N,N'-Bis-(1-cyclohexylethyl)-2,3-dimethylsuccino-diamide. White solid, m.p. > $300{ }^{\circ} \mathrm{C}$. MS $(70 \mathrm{eV}) \mathrm{m} / \mathrm{z}$ (\%): 365 (1.4) $\left[\mathrm{M}^{+}+1\right], 364$ (3.2) $\left[\mathrm{M}^{+}\right], 281$ (10), 255 (8), 239 (50), 238 (100), 128 (50).

9: 1,2-Diphthalimidoetane. White amorphous solid, m.p 242-3 ${ }^{\circ} \mathrm{C} .{ }^{1} \mathrm{H}-\mathrm{NMR}\left(300 \mathrm{MHz}, \mathrm{CDCl}_{3}, \delta\right): 4.0[4 \mathrm{H}, \mathrm{s}$, $\left(\mathrm{CH}_{2}\right)_{2}$; 7.6-7.7 (4H, m, ArH); 7.7-7.8 (4H, m, ArH). ${ }^{13} \mathrm{C}-\mathrm{NMR}\left(75 \mathrm{MHz}, \mathrm{CDCl}_{3}, \delta\right): 36.8\left(\mathrm{CH}_{2}\right), 123.3$ (CH ar.), $132.0(\mathrm{Cq}), 134.0$ (CH ar.), $168.2(\mathrm{C}=\mathrm{O})$. MS $(70 \mathrm{eV}) \mathrm{m} / \mathrm{z}$ $(\%): 320(0.9)\left[\mathrm{M}^{+}\right], 173(100), 160(92), 133(12), 105(10)$, 104 (14), 77 (22). C,H-analysis: $\mathrm{C}_{18} \mathrm{H}_{12} \mathrm{O}_{4} \mathrm{~N}_{2}$ (320.30), calculated C67.50, H3.78, N8.75, experimental C67.46, H3.87, N8.74.

10a: N-Hexylphthalimide. Colourless oil. ${ }^{1} \mathrm{H}-\mathrm{NMR}$ (300 MHz, $\left.\mathrm{CDCl}_{3}, \delta\right): 0.89\left(3 \mathrm{H}, \mathrm{t},{ }^{3} \mathrm{~J}=6.5 \mathrm{~Hz},-\mathrm{CH}_{2} \mathrm{CH}_{3}\right)$, 1.2-1.4 [6 H, m, - $\left.\left(\underline{\mathrm{CH}}_{2}\right)_{3}\right], 1.6-1.8(2 \mathrm{H}, \mathrm{m}), 3.70\left(2 \mathrm{H}, \mathrm{t},{ }^{3} \mathrm{~J}\right.$ $\left.=6.5 \mathrm{~Hz},-\mathrm{NCH}_{2}-\right), 7.7-7.8(2 \mathrm{H}, \mathrm{m}, \mathrm{ArH}), 7.8-7.9(2 \mathrm{H}, \mathrm{m}$, ArH). ${ }^{13} \mathrm{C}-\mathrm{NMR}(75 \mathrm{MHz}, \delta): 168.5(\mathrm{C}=\mathrm{O}), 133.8$ (CH ar.), $132.3(\mathrm{Cq}), 123.1$ ( $\mathrm{CH}$ ar.), $38.1\left(\mathrm{CH}_{2}\right), 31.4\left(\mathrm{CH}_{2}\right), 28.7$ $\left(\mathrm{CH}_{2}\right), 26.5\left(\mathrm{CH}_{2}\right), 22.5\left(\mathrm{CH}_{2}\right), 14.0\left(\mathrm{CH}_{3}\right) . \mathrm{IR}(\mathrm{NaCl}) \mathrm{cm}^{-1}$ : 2955, 2930, 2870, 2860 (v C-H), 1775, 1750, 1715 (v $\mathrm{C}=\mathrm{O}), 1395,1370,1270,1060,720$ ( $\delta$ aromatic). MS (70

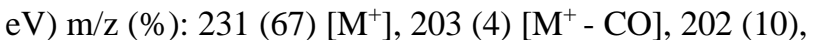
188 (7) $\left[\mathrm{M}^{+}-\mathrm{C}_{3} \mathrm{H}_{6}\right], 174$ (16), 161 (69) $\left[\mathrm{M}^{+}-\mathrm{C}_{5} \mathrm{H}_{10}\right], 160$ (100) $\left[\mathrm{M}^{+}-\mathrm{C}_{5} \mathrm{H}_{11}\right], 148$ (22).

10b: N-Methoxymethylphthalimide. White amorphous solid, m.p. $122-3{ }^{\circ} \mathrm{C}$ (Lit. ${ }^{13,15} 121-123{ }^{\circ} \mathrm{C}$ ). ${ }^{1} \mathrm{H}-\mathrm{NMR}(300$ $\left.\mathrm{MHz}, \mathrm{CDCl}_{3}, \delta\right): 3.41(3 \mathrm{H}, \mathrm{s}, \mathrm{OMe}), 5.09(2 \mathrm{H}, \mathrm{s}$, $\mathrm{NCH}_{2} \mathrm{OMe}$ ), 7.7-7.8 (2H, m, ArH), 7.9-8.0 (2H, m, ArH). ${ }^{13} \mathrm{C}-\mathrm{NMR}$ (75 MHz, $\delta$ ): $165.8(\mathrm{C}=\mathrm{O}), 134.3$ (CH ar.), 131.8 (Cq), 123.6 (CH ar.), $68.6\left(\mathrm{NCH}_{2}\right), 57.3$ (OMe). MS (70

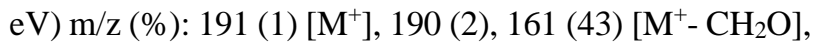

$160(100)\left[\mathrm{M}^{+}-\mathrm{OMe}\right], 148(5), 133$ (13), 104 (10), 76 (15), 50 (18).

11a: N-N-Hexyl-3-hydroxy-1-isoindolinone. White amorphous solid, m.p. $240-2{ }^{\circ} \mathrm{C}$. ${ }^{1} \mathrm{H}-\mathrm{NMR}\left(\mathrm{CDCl}_{3}, 300\right.$ $\mathrm{MHz}, \delta): 0.84\left(3 \mathrm{H}, \mathrm{t},{ }^{3} \mathrm{~J}=6.5 \mathrm{~Hz},-\mathrm{CH}_{2} \mathrm{CH}_{3}\right), 1.22-1.23(6$ $\mathrm{H}, \mathrm{m}), 1.45-1-63$ (2H, m), 3.15-3.30 (1H, m, -NCHㅍ- $)$, 3.35-3.50 (1 H, m, -NCㅌH-), $3.80\left(1 \mathrm{H}, \mathrm{d},{ }^{3} \mathrm{~J}=12 \mathrm{~Hz}\right.$, disappear with $\left.\mathrm{D}_{2} \mathrm{O}, \mathrm{OH}\right), 5.72\left(1 \mathrm{H}, \mathrm{d},{ }^{3} \mathrm{~J}=12 \mathrm{~Hz}\right.$, s after $\mathrm{D}_{2} \mathrm{O}$ addition, $\left.\mathrm{C} \underline{\mathrm{HOH}}\right)$, 7.3-7.4 (m, $\left.1 \mathrm{H}, \mathrm{ArH}\right), 7.5-7.8(\mathrm{~m}$, $3 \mathrm{H}, \mathrm{ArH}) .{ }^{13} \mathrm{C}-\mathrm{NMR}\left(\mathrm{CDCl}_{3}, 75 \mathrm{MHz}, \delta\right): 167.4(\mathrm{C}=\mathrm{O})$, $144.0(\mathrm{Cq}), 134.0$ (CH ar.), $132.0(\mathrm{CH}$ ar.), $131.6(\mathrm{Cq})$, 129.7 (CH ar.), 123.6 (CH ar.), $81.7(\mathrm{CH}), 39.1\left(\mathrm{CH}_{2}\right), 31.5$ $\left(\mathrm{CH}_{2}\right), 28.2\left(\mathrm{CH}_{2}\right), 26.7\left(\mathrm{CH}_{2}\right), 22.5\left(\mathrm{CH}_{2}\right), 14.0\left(\mathrm{CH}_{3}\right) . \mathrm{IR}$ $(\mathrm{NaCl}) \mathrm{cm}^{-1}: 3290,2955,2930,2860,1675,1620,1470$, 1445, 1420, 1060, 745, 720. MS (70 eV) m/z (\%): 233 (44) $\left[\mathrm{M}^{+}\right], 231(6)\left[\mathrm{M}^{+}-2\right], 162(41)\left[\mathrm{M}^{+}-\mathrm{C}_{5} \mathrm{H}_{11}\right], 160$ (17),

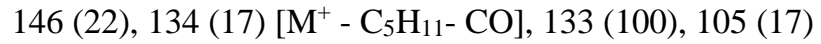
$\left[\mathrm{C}_{7} \mathrm{H}_{5} \mathrm{O}\right], 190(4)\left[\mathrm{M}^{+}-\mathrm{C}_{3} \mathrm{H}_{6}\right]$.

11b: N-Methoxymethyl-3-hydroxy-1-isoindolone. White amorphous solid, m.p. 100-101 ${ }^{\circ} \mathrm{C}$ (Lit. ${ }^{16} 103.5-$ $\left.104.5{ }^{\circ} \mathrm{C}\right) .{ }^{1} \mathrm{H}-\mathrm{NMR}\left(\mathrm{CDCl}_{3}, 300 \mathrm{MHz}, \delta\right): 3.29(3 \mathrm{H}, \mathrm{s}$, $\mathrm{OMe}), 3.74\left(1 \mathrm{H}, \mathrm{d},{ }^{3} \mathrm{~J}=10.2 \mathrm{~Hz}\right.$, disappear with $\left.\mathrm{D}_{2} \mathrm{O}, \mathrm{OH}\right)$, $4.73\left(1 \mathrm{H}, \mathrm{d},{ }^{3} \mathrm{~J}=10.5 \mathrm{~Hz},-\mathrm{NCH} \underline{\mathrm{H}}-\right), 4.81\left(1 \mathrm{H}, \mathrm{d},{ }^{3} \mathrm{~J}=10.5\right.$ $\mathrm{Hz},-\mathrm{NC} \underline{\mathrm{H}} \mathrm{H}-), 5.92\left(1 \mathrm{H}, \mathrm{d},{ }^{3} \mathrm{~J}=10.2 \mathrm{~Hz}, \mathrm{~s}\right.$ after addition of $\left.\mathrm{D}_{2} \mathrm{O},-\mathrm{C} \underline{\mathrm{HOH}}\right), 7.41-7.47$ (1 H, m, ArH), 7.53-7.61 (2 H, $\mathrm{m}, \mathrm{ArH}), 7.63-7.67$ (1 H, m, ArH). ${ }^{13} \mathrm{C}-\mathrm{NMR}\left(\mathrm{CDCl}_{3}, 75\right.$ $\mathrm{MHz}, \delta): 168.5$ (C=O), 144.1 (Cq), 132.9 (CH ar.), 130.7 (Cq), 129.8 (CH ar.), 123.7 (CH ar.), 123.6 (CH ar.), 80.5 (CH), $70.6\left(\mathrm{CH}_{2}\right), 56.3(\mathrm{OMe})$. MS (70 eV) m/z (\%): 175 (18) $\left[\mathrm{M}^{+}-\mathrm{H}_{2} \mathrm{O}\right], 163$ (28) $\left[\mathrm{M}^{+}-\mathrm{CH}_{2} \mathrm{O}\right], 162$ (22), 161 (17), 134 (20), 133 (100), 105 (42), 77 (37), 60 (56). MS (CI $\left.\mathrm{NH}_{3}\right): 211(5)\left[\mathrm{M}^{+}+\mathrm{NH}_{4}^{+}\right], 210(16)\left[\mathrm{M}^{+}+\mathrm{NH}_{3}\right], 209$ (100), 194 (6) $\left[\mathrm{M}^{+}+1\right], 193$ (2) $\left[\mathrm{M}^{+}\right], 192$ (22), 178 (31), 163 (17), 146 (47). C,H-analysis: $\mathrm{C}_{10} \mathrm{H}_{11} \mathrm{O}_{3} \mathrm{~N}$ (193.205), calculated C62.17, H5.74, N7.25, experimental C62.22, H5.81, N7.29.

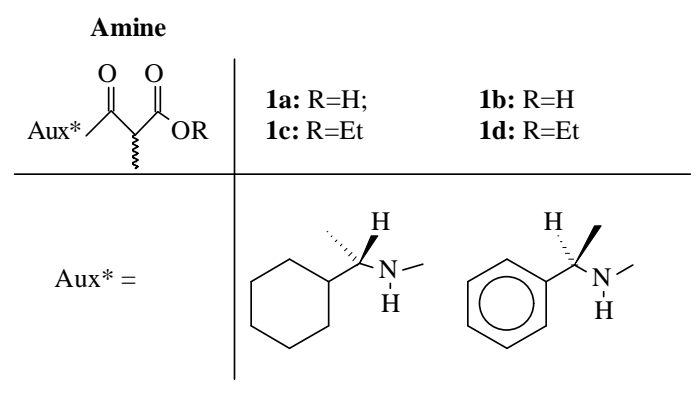

\begin{tabular}{c|cccc}
$\begin{array}{c}\text { Co-acid } \\
\mathrm{RCO}_{2} \mathrm{H}\end{array}$ & $\mathbf{2 c}$ & $\mathbf{2 d}$ & $\mathbf{2 e}$ & $\mathbf{2 f}$ \\
\hline $\mathrm{R}=$ & $-\mathrm{C}_{5} \mathrm{H}_{11}$ & $-\mathrm{CH}_{2} \mathrm{SiMe}_{3}$ & &
\end{tabular}

Figure 1. Structures of the amides (1a-b) and co-acids (2c-f). 


\section{Results and Discussion}

Two new N-substituted-2-methylmalonamic acids (1ab), with a chiral auxiliary linked through the amide function were synthesised in $\mathrm{ca} 45 \%$, non-optimised yields. They were prepared by substitution of the mono-acyl chloride derived from ethyl methylmalonic acid, with commercially available amines [(S)-(+)-1-cyclohexylethylamine and

Table 1. Results from Kolbe and mixed Kolbe electrolyses.

\begin{tabular}{|c|c|c|c|c|c|c|c|c|c|c|c|c|}
\hline Entry & $\begin{array}{l}\text { Subst } \\
(\mathrm{mM})\end{array}$ & Co-acid & $\begin{array}{c}\mathrm{Q} \\
\mathrm{F} \mathrm{mol}^{-1}\end{array}$ & $\begin{array}{c}3 \text { (MKD) } \\
(\%)\end{array}$ & de & $\begin{array}{c}4 \\
(\%)\end{array}$ & $\begin{array}{c}5+6 \\
(\%)\end{array}$ & $\begin{array}{c}8 \\
(\%)\end{array}$ & 9 & 10 & 11 & $\begin{array}{l}\text { MKD/ } \\
\text { Other }\end{array}$ \\
\hline 1 & $\begin{array}{l}\mathbf{1 a} \\
(0.5)\end{array}$ & - & 1.7 & - & - & 6 & 33 & 30 & - & - & - & 0.7 \\
\hline 2 & $\begin{array}{c}\mathbf{1 a} \\
(0.5)\end{array}$ & $\begin{array}{l}2 c \\
(5)\end{array}$ & 1.1 & $\begin{array}{c}3 \mathrm{a}, \\
\mathrm{R}=\mathrm{C}_{5} \mathrm{H}_{11} \\
56\end{array}$ & $1.3 / 1$ & 5.5 & 23.7 & traces & - & - & - & 1.9 \\
\hline 3 & $\begin{array}{l}\mathbf{1 b} \\
(1)\end{array}$ & $\begin{array}{c}\mathbf{2 c} \\
(10)\end{array}$ & 1.4 & $\begin{array}{c}3 \mathrm{~b}, \\
\mathrm{R}=\mathrm{C}_{5} \mathrm{H}_{11} \\
63\end{array}$ & $1.2 / 1$ & 6.4 & 20 & traces & - & - & - & 2.3 \\
\hline 4 & $\begin{array}{c}\mathbf{1 a} \\
(0,5)\end{array}$ & $\begin{array}{l}\mathbf{2 d} \\
(5)\end{array}$ & 1.4 & $\begin{array}{c}3 \mathrm{a}, \\
\mathrm{R}=\mathrm{CH}_{2} \mathrm{SiMe}_{3} \\
56\end{array}$ & $1.1 / 1$ & 2.7 & 16.4 & 1.8 & - & - & - & 2.7 \\
\hline 5 & $\begin{array}{c}\mathbf{1 b} \\
(0.5)\end{array}$ & $\begin{array}{l}\mathbf{2 f} \\
(5)\end{array}$ & 1.4 & $\begin{array}{c}3 \mathrm{~b}, \\
\mathrm{R}=\mathrm{CH}_{2} \mathrm{POOEt} t_{2} \\
\text { Traces }\end{array}$ & - & - & - & - & - & - & - & - \\
\hline 6 & $\begin{array}{c}\mathbf{1 b} \\
(2.5)\end{array}$ & $\begin{array}{c}2 \mathbf{e} \\
(0.5)\end{array}$ & 1.4 & - & - & - & - & - & 4.5 & $\begin{array}{l}10 \mathrm{~b} \\
27.3\end{array}$ & $\begin{array}{l}11 \mathrm{~b} \\
12.7\end{array}$ & 0.1 \\
\hline 7 & - & $\begin{array}{l}\mathbf{2 e} \\
(5)\end{array}$ & 1.3 & - & - & - & - & - & 5 & $\begin{array}{c}10 \mathbf{b} \\
28\end{array}$ & $\begin{array}{c}\mathbf{1 1 b} \\
15\end{array}$ & 0.1 \\
\hline 8 & $\begin{array}{l}\mathbf{2 f} \\
(1)\end{array}$ & $\begin{array}{c}\mathbf{2 c} \\
(10)\end{array}$ & 1.6 & - & - & - & - & - & 12 & $\begin{array}{c}\mathbf{1 0 a} \\
12\end{array}$ & $\begin{array}{c}\mathbf{1 1 a} \\
14.6 \\
11 \mathrm{~b} \\
41 \\
\end{array}$ & 0.6 \\
\hline
\end{tabular}

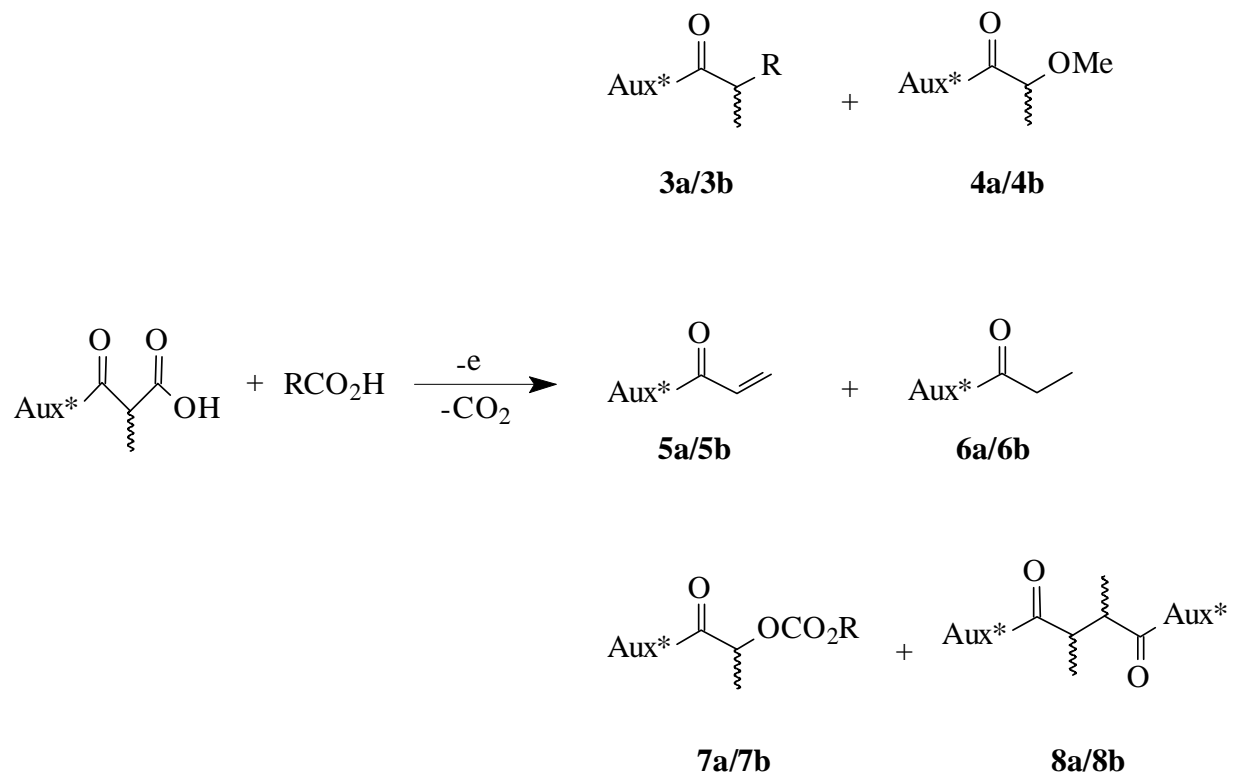

Scheme 2. Products of mixed Kolbe electrolysis: Kolbe products (K) 3a-b (mixed Kolbe dimer), desired product and 8a-b (symmetrical dimer); non Kolbe products (nK): 4a-b: Hofer-Moest products; $\mathbf{5 a - b}, \mathbf{6 a - b}$ : disproportionation products; 7a-b: esterification by starting carboxylates. 


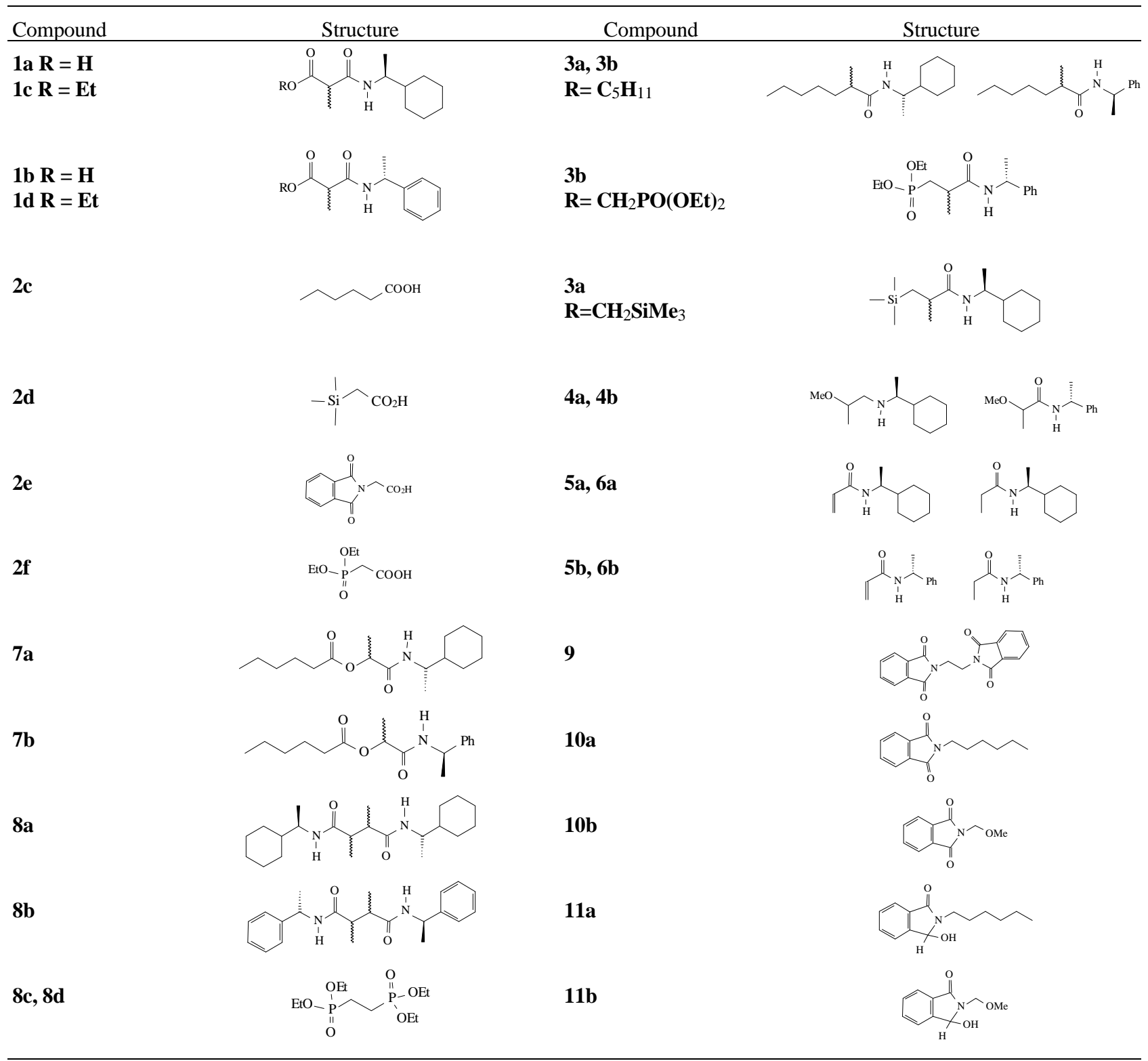

Figure 2. Structures of starting materials and reaction products.

(R)-(+)-1-phenylethylamine)], in the presence of triethylamine in absolute ether. This reaction was followed by hydrolysis of the ester group from 1c and 1d, leading to $\mathbf{1 a - b}$, as diastereomeric mixtures (Figs. 1 and 2). They were submitted as such to mixed Kolbe electrolyses, using several co-acids 2c-f (Fig. 1) (Table 1). The results from the several performed electrolyses are shown in Table 1 and summarised in Scheme 2. Figure 2 shows the structures of the starting materials and final compounds.

Mixed Kolbe dimers (MKD) (3a-b) were obtained in good yields (56 to 63\%), in the presence of hexanoic acid (2c), together with non Kolbe (nK) products, mainly from disproportionation $(\mathbf{5 a}-\mathbf{b}, \mathbf{6 a - b})$ and solvolysis (4a-b) (Table 1, entries 2,3). Hexanoate derivatives (7a-b), evidenced by $\mathrm{GC} / \mathrm{MS}$ techniques, were also present in minute amounts (not included in Table 1). Symmetrical dimers (K) (8a-b), expected Kolbe products from electrolysis in the absence of co-acids ${ }^{1}$ (Scheme 3 ) (Table 1, entry 1), and oxidation on the nitrogen of the amide, furnishing the corresponding $\mathrm{N}$-alkylated products were also observed in a very small proportion ( $<3 \%$ ) (not included in Table 1).

The diastereomeric excesses (de), determined by gas chromatography and isolation of both diastereomers (Table 1), showed that the stereoselective induction was not high (Table 1, entries 2, 3), but acceptable and encourages further investigations with other families of acyclic chiral auxiliaries. 
Experiments in the presence of co-acids $\mathbf{2 c - f}$ were performed not only to verify the stereochemical profile of the reactions but to get useful synthetic intermediates (Table 1, entries 4-8). They were chosen due to the presence of a bulky group (2d) or to promote hydrogen bonding formation between the co-acid and the chiral amide, during the coupling step (2e-f). A new amide was obtained (3a, $R=$ $\mathrm{CH}_{2} \mathrm{SiMe}_{3}$ ), with a very impressive ratio between products from Kolbe and non-Kolbe pathways $(\mathrm{K} / \mathrm{nK}=2.7)$, but without any $d e$ improvements (Table 1, entry 4).

The coupling of the methyl malonamic acid derived radicals with the electrophilic radicals derived from 2e-f did not succeed, giving, predominantly, homocoupling from the less valuable acids present in larger amounts with traces of mixed Kolbe dimers ${ }^{17}\left[\mathbf{3 b}, \mathrm{R}=\mathrm{CH}_{2} \mathrm{PO}(\mathrm{OEt})_{2}\right]$, characterised only by MS (Table 1, entry 5).

Phthaloylglycine (2e) deserved a detailed examination. It was interesting as a protected aminoacid, with further attempts to get mixed Kolbe dimer, since previous studies dealt more with non-Kolbe conditions ${ }^{13}$ and symmetrical dimerization ${ }^{18}$. Kolbe electrolysis of 2e alone (Table 1, entry 7) furnished the dimer 9, N-methoxymethyl- phthalimide (10b) $)^{13,15}$ and N-methoxymethylhydroxylactam (11b) (K/nK 0.1) (Scheme 4) ${ }^{16}$.

The unexpected presence of $\mathbf{1 1}$ can be explained by cathodic reduction of $\mathbf{1 0}$, a consequence of polarity inversion to avoid electrode passivation. Reduction of the imide carbonyl to hydroxylactams has also been accomplished in the presence of metal hydrides and amalgams ${ }^{19,20}$.

Results from the mixed Kolbe reaction of $\mathbf{2 e}$ and $\mathbf{1 b}$ (Table 1, entry 6) as well as with $\mathbf{1 a}$ (not shown) showed complete absence of MKD (3), the malonamic acids (1a and 1b) being recovered unchanged. The reason for the failure of the above mentioned radical coupling could be the similar nature of the radicals, both electrophilic, due to the presence of electron-withdrawing substituents. Differences in the acidity of the original acids as well as Pt electrode preferential adsorption of the more abundant carboxylate could be also used to explain the experimental behaviour. The malonamides, present in smaller quantities in relation to the less valuable co-acids, might have been kept far from the electrode surface. Their oxidative decarboxylation would be avoided and homocoupling of co-acids would predominate. In cases where the values of $\mathrm{pk}_{\mathrm{a}}$<smiles>C[C@H](C([14CH3])=O)C(=O)O</smiles>

$1 \mathbf{a}$

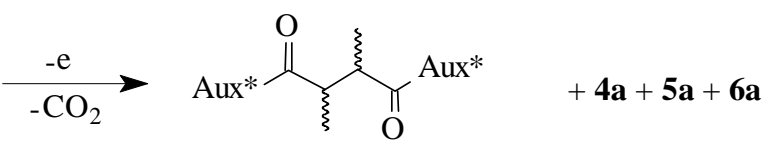

8a<smiles>CCOP(=O)(CCOP(=O)(CC(=O)O)OCC)CCP(=O)(OCC)OCC</smiles>

Scheme 3. Products of Kolbe electrolysis in the absence of co-acid. 8a: simple dimer, 4a, 5a and 6a: side products from disproportionation and Hofer-Moest reaction. 8c: symmetrical dimer from $\mathbf{2 f}$.

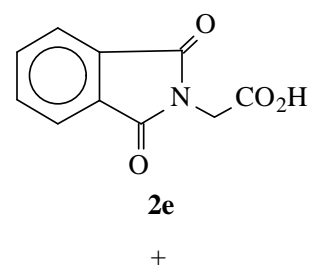

$\mathrm{RCO}_{2} \mathrm{H}$
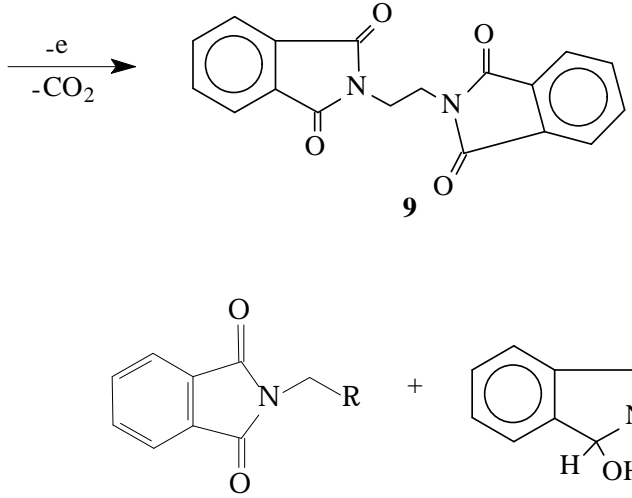

10a $\mathrm{R}=\mathrm{C}_{5} \mathrm{H}_{11}$; $10 \mathrm{~b} \mathrm{R}=\mathrm{OMe}$

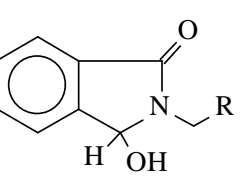

11a $\mathrm{R}=\mathrm{C}_{5} \mathrm{H}_{11}$

$11 \mathrm{~b} \mathrm{R}=\mathrm{OMe}$ 
differ strongly, a complete neutralisation is recommended, in spite of the expected lower selectivity ${ }^{1}$. Alternatively, sequential and continuous addition of one of the acids (more acidic over an excess of the weaker acid) may lead to successful cross-coupling ${ }^{1}$. Preferential adsorption of one of the carboxylates, especially the less-valuable one, onto the electrode can also be avoided by a similar strategy. Attempts (not shown in Table 1) in those directions were tried in the case of $\mathbf{1 a} / \mathbf{1} \mathbf{b}$ with $\mathbf{2 e}$, but in no circumstance, was an increase of the expected MKD evidenced.

To allow comparison, mainly with respect to reactivity and differences of acidity, $2 \mathbf{e}$ was electrolysed in the presence of 2c (Table 1, entry 8). The expected mixed Kolbe dimer (10a) was present, as well as an N-hexylhydroxylactam (11a), along with 9 and 11b. In spite of the low $\mathrm{K} / \mathrm{nK}$, there was an expressive increase on Kolbe pathway. This result also shows that the electrophilic imide-derived radical reacts easier with the nucleophilic pentyl radical. The presence of the mixed Kolbe dimer (10a, 11a), in spite of the low yield, is interesting and demonstrated the feasibility of the use of phthalic anhydride to protect the $\mathrm{N}$ functions, decreasing the amount of non-Kolbe products. Hydroxylactams obtained probably through reduction of adsorbed species due to the applied technique of reversal of polarity are useful intermediates in organic synthesis ${ }^{19,21}$.

The combined results showed that mixed Kolbe dimers are favourably obtained when the radicals have opposite reactivity (Table 1 , entries $2,3,4$ ), for instance, the high yield coupling of the highly nucleophilic pentyl or silylsubstituted radicals (derived from $\mathbf{2 c}$ and $\mathbf{2 d}$, respectively), used in excess, with the electrophilic amide-substituted ones. In this case, yields closer to the statistical ones can be obtained. Electrophilic radicals can couple between themselves, but, to a much lower extent.

Concerning the stereochemical course of the reactions, the low observed diastereoselectivity can be explained by the not yet optimal chiral auxiliaries, by their secondary amide nature and the early transition state of the coupling reaction. Increase of the volume of the malonic acid substituent, use of bulkier nucleophilic co-acids, as tried before $^{9}$ and amide $\mathrm{N}$-alkylation would be relevant for the improvement in mixed Kolbe electrolysis using $\mathbf{1 a}$ and $\mathbf{1 b .}$

\section{Acknowledgments}

The authors acknowledge financial support from CNPq, RHAE/QF and PADCT. The authors wish to thank Nivaldo A. Soares for helpful discussions.

\section{References}

1. Schäfer, H-J. Top. Curr. Chem. 1990, 152, 91.

2. Schierle, K.; Hopke, J.; Niedt, M-L.; Boland, W.; Steckhan, E. Tetrahedron Lett. 1996, 37, 8715.

3. Matzeit, A.; Schäfer, H. J.; Amatore, C. Synthesis 1995, 1432.

4. Hiebl, J.; Blanka, M.; Guttman, A.; Kollmann, H.; Leitner, K.; Mayrhofer, G.; Rovenszky, F.; Winkler, K. Tetrahedron 1998, 54, 2059.

5. Degner, D. In Top. Curr. Chemi. 1988, 148, 24.

6. Eberson, L.; Nilsson, M. J. Chem. Soc. Chem. Commun. 1992, 1041.

7. Porter, N.A.; Giese, B.; Curran, D.P. Acc. Chem. Res. 1991, 24, 296.

8. Smadja, W. Synlett. 1994, 1.

9. Klotz-Berendes, B.; Schäfer, H.J.; Grehl, M.; Fröhlich, R. Angew. Chem. Int. Ed. Engl. 1995, 34, 189.

10. Weinges, K.; Gries, K.; StemmLe, B.; Schrank, W. Chem. Ber. 1977, 110, 2098.

11. Bringmann, G.; Geisler, J.P. Synthesis 1989, 608.

12. Spenckenbach, B.; Bisel, P.; Frahm, A.W. Synthesis 1997, 1325.

13. Thomas, H.G.; Kessel, St. Chem. Ber. 1988, 121, 1575.

14. Papadoulos, A.; Lewall, B.; Steckhan, E.; Ginzel, K-D.; Knoch, F.; Niger, M. Tetrahedron 1991, 47, 563.

15. Beilsteins Handbuch der organischen Chemie, E III/IV, vol. 21; Springer Verlag: New York, 1979, pp. 5108

16. Kondo, Y.; Witkop, B. J. Org. Chem. 1968, 33, 206212

17. Lubbers, T. Doctorate thesis, University of Münster, 1991.

18. Mori, K. Nippon Kagaku Zasshi 1961, 82, 1375. Apud Chem. Abst. 1962, 57, 14929e.

19. Luzzio, F.A.; O’Hara, L.C. Synth. Commun. 1990, 20, 3223.

20. Newnam, M.S. J. Org. Chem. 1961, 26, 582.

21. Vostrowsky, H.J. Top. Curr. Chem. 1983, 109, 85. 